\title{
Le rire insupportable de Rodrigo García
}

\section{Geneviève Jolly}

\section{OpenEdition}

Journals

Édition électronique

URL : http://journals.openedition.org/recherchestravaux/298

DOI : 10.4000/recherchestravaux.298

ISSN : 1969-6434

\section{Éditeur}

UGA Éditions/Université Grenoble Alpes

\section{Édition imprimée}

Date de publication : 15 octobre 2006

Pagination : 47-61

ISBN : 978-2-84310-099-4

ISSN : 0151-1874

\section{Référence électronique}

Geneviève Jolly, «Le rire insupportable de Rodrigo García », Recherches \& Travaux [En ligne], 69 | 2006, mis en ligne le 30 septembre 2008, consulté le 07 septembre 2020. URL : http://

journals.openedition.org/recherchestravaux/298; DOI : https://doi.org/10.4000/recherchestravaux 298 


\section{Le rire insupportable de Rodrigo García}

Revendiqué par son auteur comme «violent, sale et laid ${ }^{2}$ »-adjectifs exprimant davantage un refus des normes que leur sens propre-, ce théâtre indissociablement textuel et scénique ${ }^{3}$ déroute et irrite les spectateurs, mais peut également faire rire, même si c'est à contrecœur. Il repose en effet sur un humour corrosif et décapant, dont l'appréhension et la signification oscillent entre les premier et second degrés, en passant par des formes de décalage radical par rapport aux sujets évoqués. En outre, comme il multiplie les postures énonciatives ou les points de vue troublants, il devient source d'ambiguïté, voire de provocation, quand sont tournées en dérision des valeurs communément admises, ou que sont, au contraire, portées au pinacle des valeurs politiquement ou moralement «incorrectes». Cette œuvre ferait, de la sorte, naitre un rire jaune - ou une grimace - relevant de l'effroi ou du scandale, et difficilement supportable. Elle produirait l'effet inverse de ce que peut susciter une représentation euphorique et édulcorée du réel (détente ou soulagement psychique), lorsqu'il y est question d'injustice, de misère ou de torture, mais aussi de notre indifférence

I. Né à Buenos Aires (1964), R. García travaille à Madrid, depuis 1986, comme auteur, scénographe, vidéaste et metteur en scène, avec la compagnie de La Carniceria Teatro, fondée en 1989.

2. R. García, cité par P. Sourd, «La provocation selon Rodrigo García», Théâtres. Le Magazine, $\mathrm{n}^{\circ}$ II, nov. -déc. 2003 , p. 52.

3. R. García, dans une "présentation » inaugurant L'Histoire de Ronald, le clown de McDonald', précise que «ces textes sont nés parallèlement aux créations théâtrales qui portent le même nom", et que "ce ne sont pas des pièces de théâtre à mettre en scène plus tard mais des matériaux qui, dans un livre, peuvent n'avoir en apparence aucun rapport entre eux et qui ne prennent vraiment sens que dans la structure de la représentation» (traduction de C. Vasserot, Besançon, Les Solitaires intempestifs, 2003, p. 5). Propos très juste dans la mesure où les différents «matériaux» entrent en rapport de complémentarité ou de contradiction, rapports que construit lui-même le spectateur pendant le spectacle. En outre, les textes ne conservent aucune trace du travail plastique ou sonore des spectacles, lequel participe, au même titre que le texte, à ce qu'élabore la représentation. 
ou de notre complicité vis-à-vis de cette injustice, misère ou torture. D'où le fait qu' elle puisse diviser le lectorat comme le public: alors, peut-on - doit-on - ou non en rire?

\section{Ambiguïté et instabilité}

A priori, on le devrait, et le plus franchement du monde, tant ce théâtre paraît renouer avec l'une des traditions de la farce, où «le ventre et le sexe gouvernent les motivations" de personnages dont les «ambitions vont rarement au-delà d'un bon plat de tripes ou d'un phallus de belle taille ${ }^{4}$. De fait, et indépendamment d'un attrait pour la nourriture - j'aurai l'occasion d'y revenir -, les pièces accumulent les références au corps, au sexe et à la scatologie. Avec, par exemple, la récurrence, dans quasiment tous les textes, de termes propres, argotiques et grossiers désignant les organes génitaux ou des pratiques sexuelles; mais encore avec les rots, gaz et pets sur lesquels médite le personnage de Ronald, "clown de McDonald's", ou la mésaventure pour le moins cocasse évoquée dans J'ai acheté une pelle chez Ikea pour creuser ma tombe:

Je suis dans le bureau d'un client important. On me voit entrer aux toilettes. J'ai la chiasse, j'ai la trouille, ça fait des semaines que je ne dors pas. Je chie tout ce que j'ai à l'intérieur. Et avec la puissance d'une lance d'incendie.

Il n'y a pas assez de papier pour nettoyer toute cette merde, le sol recouvert de merde, les murs, le plafond. Je dois utiliser les serviettes pour nettoyer la merde, puis les faire disparaître par la fenêtre. Dilemme: qu'est-ce qui est pire, puisqu'on m'a vu entrer dans les toilettes? Laisser les toilettes propres et passer pour un voleur de serviettes, ou bien laisser de la merde partout?5

Le farcesque relève d'une obscénité liée à l'impudeur et au registre de langue, mais encore d'un dérapage vers l'absurde («la puissance d'une lance d'incendie») et l'outrance ("le sol», «les murs, le plafond»); et de la pirouette que propose une conclusion parodiant une délibération tragique, sous forme de «dilemme» burlesque: "passer pour un voleur de serviettes, ou bien laisser de la merde partout». Le rire est donc potentiellement de mise, mais, compte tenu du caractère intime et obscène du propos - renforcé par la première personne du singulier -, celui-ci ne risque-t-il pas de se trouver tout aussi potentiellement sapé? Un rire franc et libérateur suppose la distanciation et une solidarité des rieurs n'étant pas des plus évidentes pour des spectateurs

4. B. Faivre, «Farce», Dictionnaire encyclopédique du théâtre (sous la dir. de M. Corvin), Paris, Bordas, I991, p. 321.

5. R. García, J'ai acheté une pelle chez Ikea pour creuser ma tombe, pièce suivant L'Histoire de Ronald, le clown de McDonald's, traduction de C. Vasserot, Besançon, Les Solitaires intempestifs, 2003, p. 45. 
découvrant ce texte projeté sur écran, au Théâtre national de Strasbourg ${ }^{6}$, par exemple. Première lézarde pouvant s'immiscer au sein de la collectivité formée par un public.

En outre, ce théâtre réinvente une autre manière de tromper ou de «farcer ", non plus le personnage, mais le spectateur, et il n'est pas certain que celui-ci apprécie toujours la plaisanterie. La tromperie - ou manipulation - passe d'abord par des effets de contraste entre les registres émotionnels, et oblige le public à se positionner différemment par rapport à ce qu'il peut voir et entendre, d'où une diversité de réactions possibles. Ainsi, les représentations peuvent aller de l'inhabituel (cuisiner en scène ${ }^{7}$ ) à la farce potache (jouer avec de la nourriture ${ }^{8}$ ) ou gore (des cartes bancaires censées s'enfoncer dans la tête ou dans une jambe de comédien ${ }^{9}$ ), mais également s'en prendre à certains usages: mettre à sac l'espace scénique, et notamment y cracher ou y uriner ${ }^{\mathrm{rO}}$.

La non-linéarité, la disjonction sont, du reste, inscrites dans la mise en pages des pièces publiées, mais elles sont aussi les principes régissant une mise en scène pouvant en cela emprunter à la performance. En effet, les successives séquences plus ou moins autonomes font tour à tour appel, pour ce qui concerne les comédiens, aux jeu, mime ou profération frontale, comme au chant ou à la chorégraphie. Elles élaborent des images scéniques renvoyant à la publicité, la bande dessinée, la sculpture ou la peinture, ou consistent en photographies, films ou projections de textes sur écran. L'expérience déstabilisante que vit le spectateur relève d'une forme de "bombardement» d'images, de paroles ou de musiques qui, de surcroît, mettent elles-mêmes en œuvre la manipulation, parce qu'elles déjouent presque systématiquement les attentes.

Ainsi, les "textes improvisés" qui, dans Compré una pala en Ikea para cavar mi tumba, correspondent à une succession de fêtes de Noël (I997200I), évoquées par les comédiens, s'en prennent au caractère festif et familial traditionnellement associé à ce mythe religieux et païen. Se met en place un processus répétitif (des incidents créent à chaque fois le désaccord ou l'ennui) que reconduit encore autrement la mise en scène: pour chaque «Noël», le

6. R. García, Compré una pala en Ikea para cavar mi tumba, mise en scène de l'auteur programmée (version originale surtitrée ou projetée sur écran en français) au Théâtre national de Strasbourg, en 2003.

7. R. García, pour Notes de cuisine: « imagine une table où l'on pourrait cuisiner pour de vrai, avec des brûleurs, des ustensiles, des légumes, de la viande, de l'huile et tout ce dont chaque comédien aura besoin - il est important que la femme ne cuisine pas - pour préparer le plat de son choix" (Notes de cuisine, traduction de C. Vasserot, Besançon, Solitaires intempestifs, 200I, p. 7).

8. R. García, Compré una pala en Ikea para cavar mi tumba, op. cit.

9. Ibid.

Io. R. García, Jardineria humana, mise en scène de l'auteur programmée (version originale surtitrée) par Le Maillon - Théâtre de Strasbourg, 2003. 
personnage de l'enfant s'exerce à lancer, le plus loin possible, un coq (ou une dinde) de Noël attaché à une corde. Mais ce n'est que pour mieux le remettre en cause par la suite: le volatile explose avec fracas - et éclats dont profitent les premiers rangs de la salle -, et un magnifique chant lyrique, interprété en scène a capella, se fait entendre. On aura saisi que rien ne doit jamais durablement se mettre en place, et que la passivité et la tranquillité du public ne sont guère requises; ce à quoi contribue tout particulièrement le parti pris du contrepoint ou de la rupture.

La parole prend d'ailleurs en charge cette déstabilisation des spectateurs, en faisant surgir des postures énonciatives et des formes d'adresse (avec «je», "tu», «on» ou "nous») qui ne s'associent pas nécessairement à des personnages identifiables - d'où les projections de textes sur écran. Or, même lorsqu'il s'agit de paroles de personnage, celles-ci peuvent être privées de cohérence, comme dans le cas d'une succession de répliques reposant sur «la répétition et la négation, procédés qui annulent la progression des échanges ${ }^{\mathrm{II}}$ ». L'ambiguïté, voire la franche provocation, peuvent encore provenir de l'incongruité du positionnement d'un unique locuteur par rapport à son discours. Ainsi, le "personnage» que Notes de cuisine désigne par « $\mathrm{M}$ » prend en charge un monologue présentant initialement une situation banale (un père convoqué par une directrice d'école au sujet de son fils), amenant ensuite une série de révélations moins banales, justifiant selon la meilleure foi du monde l'alcoolisme de l'enfant ( $\mathrm{M}$ feint de s'adresser à la directrice) :

Et vous voulez que je laisse

Mon gosse à l'école

Sans une goutte d'alcool?

Trop risqué

Livré à des gens comme vous

C'est de l'inconscience

Je préfere l'emmener au bar

$\grave{A}$ sept heures

Un vermouth

Des olives vertes

Un vermouth

Des olives noires

De l'anisette avec des glaçons

Et zou

En classe $^{\mathrm{I2}}$

II. S. Le Pors et A. Martinez, "Rodrigo García, Notes de cuisine», dans Nouveaux territoires $d u$ dialogue (sous la dir. de J.-P. Ryngaert, assisté de J. Danan et S. Lepors), Arles-Paris, Actes Sud-Papiers et CNSAD, 2005 , p. I57.

I2. R. García, Notes de cuisine, op. cit., p. 28. 
La provocation relève de la pseudo-absence de distance d'une parole apparemment ordinaire, prenant pourtant à contre-pied des valeurs sociales (famille, éducation et scolarité), pour dérationnaliser ce qui devrait précisément tenir de la raison: se réjouir qu'un enfant aille à l'école; veiller sur sa santé. On frise l'absurde ou le grotesque, et, comme la formulation se fait légère et plaisante, prenant même la forme d'une comptine, le propos peut installer une distanciation et une solidarité des rieurs dans la salle. À l'inverse, si le discours devient grossier, dépréciatif et culpabilisant vis-à-vis d'une partie du public, il dénie pour celle-ci toute distance, et met à mal l'esprit de solidarité. Dans Jardinage humain, par exemple, la subversion des valeurs que met en ouvre l'instance de parole anonyme pourrait susciter, à la place du rire, une hostilité des mères ou des pères de famille:

On ne met pas des vies au monde, on met des esclaves au monde. Tu as toujours été victime des autres, alors tu mets des enfants au monde pour voir si tu as plus de chance avec eux. [...]

C'est pour ça qu'on baise et qu'on fait naître des esclaves. Par honte, par vengeance, par rancœur et par une nécessité primaire d'affirmation de soi : si tu domines un enfant, tu te sens important, bordel.

Je persiste à dire que le plus pratique est d'avoir chez soi un animal de compagnie. $^{\mathrm{I3}}$

Le principe reste le même, prendre le contre-pied de ce que la société légitime: donner la vie par générosité et ne pas confondre un enfant et un animal. Mais comme cette prise de position, ouvertement cynique et grossière, s'accompagne de l'obligation - au moins pour ceux qui ont des enfants - de se sentir concernés, elle peut rendre caduque l'existence du rire pour une partie de la salle. En revanche, et dans la même pièce, les «trois listes» faisant suite à cet extrait favorisent a priori l'empathie de l'ensemble de la salle, mais elles ne sont pas, cette fois, censées susciter un quelconque rire, lorsqu'il est fait référence à la dictature ayant sévi en Argentine, sous la forme d'un titre mentionnant explicitement les termes «répression» et «torture», et annonçant que certains responsables, nommés par la suite, ont été officiellement graciés:

LISTE DES PERSONNES AYANT COMMIS DES ACTES DE RÉPRESSION ET DE TORTURE ENTRE I976 ET 1983 EN Argentine et Graciées Par Carlos Menem EN I990 ${ }^{\text {I4 }}$

L'un des enjeux de ce théâtre consiste donc à dire publiquement, dans une salle de spectacle, ce qui ne se dit pas ouvertement, sur un plan politique ou moral, tout en multipliant les occasions de faire surgir un rire pouvant rassembler ou diviser le public - autre moyen de le tromper ou de le «farcer»,

I3. R. García, Jardinage humain, traduction de C. Vasserot, Besançon, Les Solitaires intempestifs, 2003, p. I3.

I4. Ibid., p. 4I-45. Il s'agit de cent quarante et un patronymes. 
mais de façon plus cruelle. En cela, ce théâtre le fait «travailler», le soumet à rude épreuve, parce que la communauté des rieurs va se déplacer, et ne pas toujours se trouver constituée des mêmes personnes, ce qui divise encore autrement la salle. En outre, et comme certains moments ne sont ni comiques ni humoristiques, et renvoient à des événements ou faits sérieux voire terribles, le rire devient problématique, entre dans un paradigme qui n'est pas celui du divertissement ou du soulagement physique et psychique, ce qui peut encore produire une autre forme de division.

\section{Incertitude et effroi}

Je rapprocherais volontiers cette subversion affectant le rire de l'analyse que fait Mireille Losco du théâtre de George Tabori:

Ce qui est en jeu, c'est la défaite de toute fixité non seulement dramaturgique - celle des genres - mais aussi idéologique, car la critique de la forme par la mobilité infinie du jeu désigne l'impossibilité de proposer un regard stable sur l'Histoire. ${ }^{\mathrm{IS}}$

L'œuvre de Rodrigo García emprunte aussi à la rhapsodie ${ }^{16}$, et se faisant labile, elle confronte le spectateur à une absence de "fixité» ou "mobilité infinie», voire la contradiction ou l'aporie, et ne propose pas de repères clairement interprétables; ce qui peut indisposer ou heurter, concernant certaines questions morales ou politiques. Comme en témoigne une déclaration d'intention de l'artiste, ce théâtre "répond violemment à la violence qu['il] ressen[t] tout simplement autour de [lui $]^{17}$ », et il paraît logique que, par un effet retour, une forme de violence ait à voir avec le rire que suscitent les spectacles. Dans la mesure où le comique n'y est pas aussi consensuel que peut l'être celui de la télévision, il n'est plus réductible à la définition d'Aristote, c'est-à-dire «un défaut ou une laideur qui ne causent ni douleur ni destruction $»^{18}$. Dans cet univers, il s'agit bien de "défaut» ou de "laideur", mais qui ne relèvent pas du consensus, et peuvent induire "douleur» et "destruction".

Le texte peut innocemment renvoyer à un quotidien sur lequel l'auteur porte un regard déformant ou grossissant, de façon à «étrangéiser» le nôtre,

I5. M. Losco, "Le comique comme détour: George Tabori», Études théâtrales, n 24-25, (Écritures dramatiques contemporaines 1980-2000. L'avenir d'une crise, sous la dir. de J. Danan et J.-P. Ryngaert), Louvain-la-Neuve, 2002, p. I22.

I6. Voir C. Hersant et H. Kunst, "Rhapsodie» (Lexique du drame moderne et contemporain, Belfort, Circé Poche, 2005, p. I83-187) pour l'analyse de ce concept de J.-P. Sarrazac, et le renvoi à plusieurs de ses travaux, dont L'Avenir du drame (Lausanne, L'Aire, 1981, et réédition Circé Poche, 1999).

17. R. García, cité par P. Sourd, «La provocation selon Rodrigo García», op. cit., p. 52.

I8. Aristote, La Poétique, traduction et notes de R. Dupont-Roc et J. Lallot, Paris, Seuil, I980, p. 49 (49 a 32-36). 
et à ironiser sur des pratiques de consommation. Ainsi en va-t-il des véhicules "monospaces", conçus selon lui "pour des gens qui, enfants, rêvaient d'être chauffeurs de bus, et à qui, une fois adultes, la chance a fini par sourire ${ }^{19}$ ". Cette plaisanterie repose sur une analogie "forcée», transformant une commodité (utile aux familles nombreuses) en un fantasme ou caprice puéril, et tout en feignant de s'en réjouir. De tels jeux de langage peuvent faire songer à des "brèves de comptoir", mais comme ils se répètent à longueur de textes et donc de spectacles, ils finissent par brosser un portrait-charge de spectateurs nécessairement concernés à un moment ou à un autre. Jardinage humain, par exemple, évoque des comportements fétichistes vis-à-vis du téléphone portable, et bêtifiants à l'égard d'animaux domestiques, et, comme l'instance de parole ne s'épargne pas (concernant son téléphone portable ou son chien), on ne peut guère lui reprocher de s'exclure du champ de ses critiques, et l'accuser de mauvaise foi.

Néanmoins, le bât blesse davantage quand ces habitudes de consommation induisent l'injustice: dans L'Histoire de Ronald, le clown de McDonald's, il s'agit de la maltraitance d'enfants issus de zones géographiques désignées, par un euphémisme quelque peu hypocrite, comme des "pays en voie de développement». Sachant que le rire se fait d'autant plus «jaune " que le propos semble neutre, ou, du moins, que le second degré et le décalage ne sont perceptibles que dans la reformulation insistante et parfois crue du propos:

Je me suis dit: soyons positif, bordel; il vaut mieux manger ça [un hamburger] que de sucer des bites.

Et un enfant en train de manger un Happy Meal n'est pas en train de sucer une bite. $\mathrm{Si}$ tu as neuf ans et que tu vis à Lisbonne, tu vas au McDonald's le dimanche. $\mathrm{Si}$ tu as neuf ans et que tu vis à Cuba, tu vas sucer la bite d'un touriste italien. $\mathrm{Si}$ tu as neuf ans et que tu vis à Bruxelles, tu vas au McDonald's le dimanche. $\mathrm{Si}$ tu vis en Bolivie, tu vas à la mine pour les Américains.

$\mathrm{Si}$ tu as neuf ans et que tu vis à Florence, tu vas au McDonald's le dimanche.

$\mathrm{Si}$ tu vis en Afrique, tu couds des ballons pour Nike.

$\mathrm{Si}$ tu as neuf ans et que tu vis à New York, tu vas au McDonald's le dimanche.

$\mathrm{Si}$ tu as neuf ans et que tu vis en Thaillande, tu dois te laisser enculer par un Australien. ${ }^{20}$

La syntaxe répétitive et symétrique des énoncés ainsi que le principe d'opposition entre pays riches et pays pauvres permettent de souligner une injustice patente, à l'œuvre dans différents pays, tout en accusant un anonyme "touriste italien» ou australien, et, nommément, les "Américains» et la firme «Nike». Outre le fait d'énumérer tout ce que les adultes peuvent infliger à des

19. R. García, J'ai acheté une pelle chez Ikea pour creuser ma tombe, op. cit., p. 44.

20. R. García, L'Histoire de Ronald, le clown de McDonald's, op. cit., p. 27. 
enfants, la mise en pages invite encore à placer sur un même plan des formes d'exploitation discutables, mais légales (recruter des enfants pour la mine), et des pratiques illégales (abus sexuel).

On comprend bien que le personnage envisage négativement le sort réservé aux enfants cubains, boliviens ou thaillandais; point de vue pouvant donc être partagé par les spectateurs, mais pouvant aussi mettre mal à l'aise, éthiquement parlant. Car cela suppose de cautionner l'affirmation initiale («soyons positif, bordel; il vaut mieux manger ça que de sucer des bites"): c'est-à-dire non seulement le langage grossier, mais également l'utilité pour des enfants de manger des hamburgers - et chaque dimanche -, alors que la pièce ne cesse de dénoncer leur qualité alimentaire, et le caractère contestable des pratiques commerciales de l'entreprise qui les vend. Seulement, ne pas accepter l'affirmation initiale revient à approuver la maltraitance des enfants dans les pays pauvres: cruel effet de double bind.

Cette mise au jour de contradictions irrésolues ou d'alternatives conflictuelles est précisément ce qui associe le rire à la «douleur » et à la "destruction ", parce que cela confronte directement à une mauvaise foi que nous rejetons sur les gouvernants, pour avoir bonne conscience, mais que Rodrigo García nous oblige à nous réapproprier: en faisant montre de l'égoïsme ou du cynisme d'Occidentaux dont les enfants sont libres et bien nourris. C'est peut-être ce qui pourrait expliquer les réactions d'agressivité que déclenchent les représentations: soupirs, toux, départs bruyants, mais, surtout, les demandes de remboursement du billet, ou les interventions interrompant le spectacle, parce que cela constitue l'indice, sinon d'un déni artistique, du moins du caractère proprement insupportable de ce rire.

L'insupportable est d'hésiter à rire, ou de le regretter a posteriori, et de se croire atteint d'une forme d' «anesthésie du rieur ${ }^{2 \mathrm{I}} »$. Parce que le théâtre relève de la sphère publique, et que tout spectateur a des voisins, même s'il s'agit d'inconnus. Plus simplement parce que, dans une salle de théâtre, le rire n'est pas purement spontané, et qu'il se trouve pour partie conditionné par les réactions de l'ensemble du public. L'insupportable tient donc au caractère effrayant voire honteux de ce rire, quand il surgit à la suite de propos adoptant le point de vue du dominant, de l'oppresseur, et affectant de culpabiliser la bonne conscience comme de déculpabiliser la mauvaise conscience (même pièce que précédemment) :

On a eu les Malouines, on a eu l'Afghanistan, on a eu Auschwitz pour apprendre une bonne fois pour toutes que quelqu'un doit tendre l'autre joue, car si personne ne tend l'autre joue, ça n'avance pas, bordel. Soit on vient au monde pour

2I. H. Bergson, Le Rire. Essai sur la signification du comique [1899], Paris, Presses universitaires de France, 1940, p. 53. 
distribuer des raclées - comme Margaret Thatcher, pour donner un nom comme ça au hasard -, soit on vient au monde pour recevoir des raclées, bordel.

Et souvent on méprise ceux qui reçoivent les raclées: on dit qu’ils sont faibles.

Non, monsieur.

Sans eux, on n'aurait pas écrit l'histoire de l'humanité.

Ils sont balèzes ces gens qui viennent au monde pour recevoir des raclées! ${ }^{22}$

Les référents initiaux sont connus de tous, guerres (Malouines, Afghanistan) et extermination des Juifs (Auschwitz), événements impliquant des périodes et des peuples identifiés, mais qu'il s'agirait d'accepter comme données humaines, rationnelles et immuables. Le traditionnel rapport dominants-dominés devient une sorte d'universel valable de tous les temps et partout dans le monde; il se voit comiquement radicalisé par l'analogie avec un rapport de force physique, mais cette fois entre individus ("distribuer» et "recevoir des raclées»); et, contre toute attente, le fait de "recevoir des raclées» devient un ironique devoir de charité chrétienne ("tendre l'autre joue»), digne d'admiration ("Ils sont balèzes»), et permettant d'écrire «l'histoire de l'humanité».

Cynisme ou gros bon sens? Des vérités sont dites, mais en forçant le raisonnement jusqu'à l'absurde, et en créant un lien de complémentarité entre la bonne et la mauvaise conscience, le bien et le mal, le juste et l'injuste, comme si tout se valait dans un monde qui va et fonctionne mal. Pour le spectateur, il n'y a plus de reconnaissance confortable suscitant une satisfaction intellectuelle, ou un sentiment de supériorité pouvant occasionner le rire, mais une mise en déroute des valeurs, et l'absence de solution, d'où une perspective quasiment tragique, alors même qu'est sous-entendu un appel au refus de cette "histoire de l'humanité». Mais cela suppose de prendre le texte et la mise en scène au second degré, ce que ne font pas toujours les spectateurs.

\section{Scandale et violence}

Lors d'une rencontre avec l'auteur-metteur en scène, à Strasbourg ${ }^{23}$, plusieurs membres du public firent des reproches de violence à l'égard d'un spectacle n'en comportant pas, Compré una pala en Ikea para cavar mi tumba. Il s'agissait notamment du moment où le jeune comédien jouant le rôle de l'enfant a une assiette de soupe attachée au visage par du scotch, et souffle sur cette soupe qui se répand sur le sol; et, par ailleurs, du tableau final dans lequel deux acteurs adultes - les parents de l'enfant - feignent d' «ingérer» de la nourriture (pâtes et salade, notamment) par l'extrémité postérieure de leur corps, à ce moment-là dénudée. La violence dont il était question concernait

22. R. García, L'Histoire de Ronald, le clown de McDonald's, op. cit., p. 20.

23. Rencontre organisée à l'initiative du Théâtre national de Strasbourg, dans une librairie de Strasbourg, et concernant Compré una pala en Ikea para cavar mi tumba, op. cit., en 2003. 
les comédiens "obligés» de réaliser des actions humiliantes, voire contraints de «souffrir» physiquement.

Je résume une réponse insistant sur le fait qu'il s'agissait de théâtre, que les acteurs - l'adolescent comme les adultes - prenaient beaucoup de plaisir à jouer ainsi avec la nourriture, et que le tableau final était une proposition des comédiens en répétition. Par ailleurs ces images dénuées de violence lui semblaient susceptibles de renvoyer à des réalités: le fait que tous les enfants jouent avec la nourriture, et que, selon lui, les adultes continuent à le faire, mais sans s'en rendre compte et sous la forme d'un gaspillage journalier, alors qu'une partie du monde souffre de la faim. J'ajoute que ce jeu avec la nourriture constituait précisément le fil rouge du spectacle, avec un plateau finalement jonché de lait, de corn flakes, de mayonnaise, de ketchup, de soupe, etc., mais réalisant aussi une image plastique reposant sur le contraste des couleurs et des matières.

Certains spectateurs manqueraient-ils à ce point d'humour ou de bonne foi pour confondre fiction et réalité à propos d'un spectacle qui, en cela, relevait plutôt du cabaret ou d'une "enfarinade"? Mais sans doute ont-ils projeté une part d'eux-mêmes sur ce spectacle (ce qu'eux-mêmes ne supporteraient pas de faire), et il est vrai que proposer de la vraie nourriture, et donc des odeurs et des matériaux réels, peut éventuellement susciter l'identification, ou, du moins, une forte implication - ne serait-ce qu'au moment où le volatile explose! Il reste qu'un spectacle n'est pas censé être regardé autrement que comme un spectacle, et je m'appuierai, cette fois de façon plus exacte, sur les propos de l'artiste:

Je ne suis pas un performer du réel, je travaille sur des symboles. Il s'agit de théâtre. Mon univers n'est pas de ceux où l'on fait couler le sang [...]. Il s'agit d'images, et elles servent un propos. Si elles correspondaient à des faits réels, elles ne seraient qu'une suite d'actions sinistres et voyeuristes qui ne poseraient aucune question au public. $^{24}$

Dans ce spectacle, les images ou symboles posent effectivement des questions au public sur le gaspillage de denrées alimentaires, même si elles n'apportent pas les réponses que certains spectateurs voudraient y trouver, mais, précisément, parce que ce théâtre installe l'ambiguïté et donc l'inquiétude. En revanche, et c'est peut-être ce qui dérange et scandalise, elles présentent un lien avec la performance, non pas parce que ces images scéniques consisteraient en «faits réels", mais parce qu'elles transgressent des interdits : répandre et donc gâcher de la nourriture sur un plateau de théâtre; s'en recouvrir le corps ou s'y vautrer, et - bien davantage - se toucher l'anus et le sexe en y frottant ladite nourriture;

24. R. García, cité par P. Sourd, «Rodrigo García: révolté hispanisant», Théâtres. Le Magazine, $\mathrm{n}^{\circ}$ 6, Paris, déc. 2002-janv. 2003, p. 61. 
cela, au su et au vu d'un public pouvant effectivement se trouver en position de voyeurisme, s'il ne s'esclaffe pas de l'insolence du spectacle.

Mais il y avait encore un autre moment intéressant, compte tenu de la réaction de la salle, celui de la « PETITE LISTE IN PROGRESS DES PRINCIPAUX ENFOIRÉS DE L'HISTOIRE DE L'HUMANITÉ ${ }^{25}$ ». Le public a visiblement (et ostensiblement) mal pris le fait que les comédiens - dont le jeune garçon, et c'est à noter parce qu'il y avait nécessairement des parents dans la salle - puissent insulter et brocarder certains de ces "enfoirés de l'histoire de l'humanité", tels Gandhi ou Nelson Mandela. Sans doute était-ce la mention de noms réellement existants, accompagnée de la projection de photographies de ces personnalités, qui, là encore, semblait pouvoir faire de cette scène une réalité appelant une réaction, et non ce qu'elle était: un moment de théâtre.

Dans la fiction, il s'agit d'un jeu familial consistant à se défouler sur une liste de personnalités «responsables» de divers «travers» moraux et sociaux. Alors, certes, la présence insistante d'insultes - à dix-huit reprises, même si ce ne sont pas toujours les mêmes -, et parfois proférées «comme» en direction du public, pouvait indisposer, mais ce qui faisait réagir était encore d'une autre nature. En effet, si certains spectateurs voulaient bien rire d'Elvis Presley qualifié de "drogué" et d' "obèse», ou de Sigmund Freud, "responsable» de l'existence de nos névroses et psychoses, ils ne le pouvaient plus avec, par exemple, Oscar Wilde, vilipendé comme homosexuel, ou Nelson Mandela, accusé d'être «nègre» - le tout étant, je le rappelle, assaisonné d'injures et de gestes obscènes. Se mettait alors en place, au sein du public, et vis-à-vis de ces personnalités, une forme de jugement ou de "classement» insensés - car à quel titre? - pouvant «autoriser" ou non le rire.

Or, c'est précisément l'absence de hiérarchie ou de rapport entre les différents noms qui fait l'intérêt de cette liste, parce qu'elle confronte le spectateur à un total décalage avec la réalité - laquelle n'est pourtant jamais que celle que perçoit chacun. Car cette liste affiche et souligne ainsi la distance à prendre, en tournant indifféremment en dérision chacune des personnalités. On retrouve le principe déjà mentionné d'une mise en déroute de valeurs qui peuvent être celles des spectateurs: car s'il est consensuel de ne pas se moquer des Médecins du Monde, qu'en est-il pour l'apôtre saint Jacques ou Che Guevara? Là encore, et compte tenu de la disparité des personnalités, le public se trouve divisé: sur le plan moral, politique, ou selon ses croyances religieuses. Tous ne rient pas au même moment et

25. R. García, J'ai acheté une pelle chez Ikea pour creuser ma tombe, op. cit. Figurent, sur cette liste ou ce classement: «Elvis Presley / Marilyn Monroe / Paul Bocuse / Andy Warhol / Pablo Picasso / Sigmund Freud / L'apôtre saint Jacques / Pier Paolo Pasolini / le Mahatma Gandhi / Nelson Mandela / Janis Joplin / Oscar Wilde / Médecins du Monde / Freddy Mercury / Jorge Luis Borges / le Che Guevara / John Lennon / Diego Maradona» (p. 57). 
des mêmes choses, et, alors que certains rient de ce qui ne fait pas rire leurs voisins, ces derniers sont néanmoins confrontés à leurs rires, et réciproquement.

La virulence des réactions indiquait clairement le refus d'apprécier le second degré de cette mise en scène, mais elle était également l'indice du scandale qui consiste à faire rire de brocards concernant notamment des personnalités entrées dans la légende, voire sacralisées. Et pourtant, l'ensemble de ces brocards visait en l'occurrence à mettre à plat des formes d'hypocrisie et d'intolérance: vis-àvis de la femme, de l'homosexualité ou de la couleur de peau, par exemple. Or, dans la mesure où ces injures ou marques d'intolérance "ont été» et "sont» encore présentes ici ou là, il s'avère peut-être utile de les réentendre dans un lieu public, sur une scène de théâtre, et de se laisser aller à rire de leur non-sens, puisqu'elles entrent dans un paradigme où tout finit par se valoir.

Ce théâtre présente l'intérêt de revisiter et de renvoyer dos-à-dos des clichés et préjugés qui perdurent, même lorsqu'on les croit ou veut les croire disparus. De ce point de vue, il travaille à partir du réel, en présente une «lecture», tout en détournant ce qui est emprunté à la réalité. Son propos fonderait de la sorte une conception de l'humour "qui relève plus d'un "travail" sur le réel que d'une imitation de ce dernier ${ }^{26}$ ». Si bien que réduire ce spectacle à une série de faits prétendument réels empêche d'avoir accès, d'une part, à ce " "travail" sur le réel»- le démontage de clichés et de préjugés intolérants -, et d'autre part, cette fois pour l'ensemble de l'œuvre, au fait de réapprendre à voir, entendre et lire, pour ne plus accepter sans broncher les images et paroles que déversent quotidiennement la publicité ou les informations de la radio et de la télévision.

La diversité - disparité - du flot d'images et de paroles que proposent ces spectacles, parce qu'elle n'impose pas un sens univoque et immédiat, mais joue sur le contrepoint et la rupture, oblige le spectateur à se (re)positionner sans cesse, et donc à adopter un comportement qui n'est pas celui qu'induisent les médias : passivité et absence d'esprit critique. De plus, la division qui peut affecter le public à l'égard du rire peut constituer le moyen de le responsabiliser: un spectateur n’a pas nécessairement à réagir comme le font ses voisins. Mais la violence est peut-être là, finalement: dans le fait de détruire ou de saper le beau - mais mensonger - consensus d'un soir, dans une salle de théâtre.

Le théâtre de Rodrigo García fait donc naître un rire ambigu, source d'effroi, voire de révolte, parce qu'il ne repose ni sur l'euphorie ni sur le consensus, et qu'il ne se réfugie pas dans le bien-pensant ou le bienséant, bien au contraire. Dans cet univers, surgissent des vérités qui ne sont pas toujours bonnes à dire, et donc à entendre, mais qu'il est salutaire d'entendre. Surgissent également

26. M. Losco, «Le comique comme détour: George Tabori», op. cit., p. I25. 
des réalités n'étant plus perçues comme telles, et dont il s'agit de prendre conscience, en public, précisément. Accepter de rire pourrait être le moyen de s'opposer aux défauts et à la laideur du monde, en reconnaissant leur part de douleur et de destruction. Accepter ce rire dérangeant ou insupportable revient alors à prendre une liberté, un risque, et donc une responsabilité (se remettre en cause), ce que tous ne semblent pas prêts à faire. Sans doute parce qu'au théâtre le spectateur n'est jamais seul, et que, pour lui, le fait de rire - comme de ne pas rire - implique le regard des autres, et, par effet de miroir, un regard sur soi-même. Voilà pourquoi ce théâtre est particulièrement méchant, "violent, sale et laid ${ }^{27}$ »! Mais voilà aussi pourquoi son auteur peut penser en tirer un constat d'échec:

J'ai pensé qu'il y avait une chance pour la poésie. / [...] lorsqu'en tant qu'artiste je sème la poésie et la confusion dans une salle de théâtre, je me comporte en vil escroc. / Mais quand, dans ma vie quotidienne, $\mathrm{j}$ 'accède à un instant de poésie, je me laisse aller sans regarder à la dépense, je m’applique à en créer un autre. / La capacité poétique est en l'homme, il faut s'y entraîner. [...] / J'ai eu un grand espoir. J'ai trouvé des forces extraordinaires pour créer sans souffler, sans me rendre compte qu'il s'agissait d'une entreprise titanesque pour un gars comme moi. Voici venu le moment de la déconvenue, du doute et du tremblement. J'ai beau chercher, je ne trouve aucun rapport entre mon ouvre et l'amélioration d'un monde malade. [...] / Eh bien, il y a un temps pour parler et un temps pour se taire. ${ }^{28}$

Ce qui est peut-être dommage.

27. R. García, cité par P. Sourd, «La provocation selon Rodrigo García», op. cit., p. 52.

28. R. García, "Ce gars-là, on ne veut plus le revoir », Mouvement, n 36-37, Paris, sept. - déc. 2005, p. 27. Ce texte traduit par C. Vasserot a été conçu pour le colloque international de Rennes, Mises en scène du monde (Besançon, Les Solitaires intempestifs, 2005). 